\title{
Spectrum of Curvature Perturbation of Multi-field Inflation with Small-Field Potential
}

\author{
Iftikhar Ahmad, Yun-Song Piao ${ }^{\dagger}$ and Cong-Feng Qiao \\ College of physical sciences, Graduate University of Chinese Academy of Sciences, \\ YuQuan Road 19A,Beijing 100049, China.
}

\begin{abstract}
In this paper, we have studied the spectrum of curvature perturbation of multi-field inflation with general small-field potential. We assume that the isocurvature perturbation may be neglected, and by using the Sasaki-Stewart formalism, we found that the spectrum may be redder or bluer than of its corresponding single field. The result depends upon the values of fields and their effective masses at the horizon-crossing time. We discuss the relevant cases.
\end{abstract}

\section{Introduction}

Inflation [1, 2, 3] naturally solves the flatness, homogeneity and monopole problems and predicts almost scale invariant density perturbations, consistent with present observations. Thus inflation has become the dominant paradigm for understanding the features of our observable universe. However, single field inflation model generally has some fine tuning problems of the parameters, such as the mass and coupling of field, and also the value of field, which renders it difficultly be realized in a realistic high energy theory.

When many fields are included, it was found that they can work cooperatively to drive a period of inflation by assisted inflation mechanism proposed by Liddle et al. [4], even if any one of those fields is not able to sustain inflation separately. The multi-field inflation model relaxes the difficulties suffered by single field inflation models, and thus may be regarded as an attractive implementation of inflation. The assisted inflation with massive fields have been discussed earlier in [5], and subsequently explored in the Kaluza-Klein model [6] and in the Randall-Sundrum model [7] where there would be a tower of mass eigenstates. Recently, there have been many interesting examples of multi-field inflation e.g. see [8], in which exponentially large number of field was required for a feasible theoretic realization of inflation. In addition, some multi-field inflation models have been also discussed in [9].

It was shown [10] that the many axion fields predicted by string vacuum can be combined and lead to a radiatively stable inflation, called N-flation. These axion fields generally have different masses, which can be very densely spaced. The spectrum of curvature perturbation of multi-field inflation with unequal masses have been discussed in [11, and then the detailed study was made by Easther and McAllister [12] for quite specific choices of initial conditions for the fields, and the numerical investigation was made by Kim and Liddle [13] for the random initial conditions. Recently, it was shown analytically in [14] that for multi-field inflation with power law potential,

*e-mail:iftikharwah@gmail.com

${ }^{\dagger}$ e-mail:yspiao@gucas.an.cn

${ }^{\ddagger}$ e-mail:qiaocf@gucas.an.cn 
the spectrum of curvature perturbation is generally redder than that of its corresponding single field. The result obtained from that with unequal massive fields is consistent with the numerical investigation of Kim and Liddle, and is also compatible with recent other studies [15, 16].

It is interesting to generalize above studies to multi-field inflation with other potentials. The small-field inflation model arises naturally from spontaneous symmetry breaking such as the original models of new inflation [17, 18, modular inflation from string theory [19] and from Pseudo Nambu-Goldstone modes (natural inflation) [20]. In this class of model, the field generally starts at near an unstable equilibrium (usually taken to be at the origin) of its potential and then rolls down along the potential to a stable minimum. Thus the potential of the smallfield models can be taken as $V(\phi)=\Lambda\left[1-(\phi / \mu)^{p}\right]$, which can be viewed as a lowest-order Taylor expansion of an arbitrary potential around the origin. A natural generalization to multiple uncoupled fields may be written as

$$
V=\sum_{i} V_{i}\left(\phi_{i}\right)=\sum_{i} \Lambda_{i}\left[1-\left(\frac{\phi_{i}}{\mu_{i}}\right)^{p}\right],
$$

where the subscript " $i$ " denotes the relevant quantities of the $i$ th field and $p$ is the same for all fields, and $\Lambda_{i}$ and $\mu_{i}$ are the parameters describing the height and tilt of potential of the $i$ th field. In this paper, we will study the spectrum of curvature perturbation of multi-field inflation with the potential (1).

This article is organized as follows; in section 2 we calculate the spectral index of curvature perturbation by using the Sasaki-Stewart formalism [25] for $p>2$ and discuss the relevant results. In section 3 we focuss on the case of $p=2$. Finally, in section 4 we summarize our conclusion.

\section{The Scalar Spectrum of Spectral Index for $p>2$}

With the potential (1), in the slow-roll approximation, we have

$$
\begin{gathered}
3 H \dot{\phi}_{i}+V_{i}^{\prime}\left(\phi_{i}\right) \simeq 0, \\
H^{2} \simeq \frac{1}{3 M_{p}^{2}} \sum_{i} V_{i}\left(\phi_{i}\right),
\end{gathered}
$$

where $H=\dot{a} / a$ is Hubble parameter, $a(t)$ is scalar factor, and $M_{p}=(8 \pi G)^{-1 / 2}$ is the Planck mass. From Eq. (2), we can obtain

$$
\frac{\dot{\phi}_{i}}{V_{i}^{\prime}\left(\phi_{i}\right)}=\frac{\dot{\phi}_{j}}{V_{j}^{\prime}\left(\phi_{j}\right)}
$$

Thus the efolding number is

$$
\begin{aligned}
N & =\int H d t=\int H \frac{d \phi_{j}}{\dot{\phi}_{j}} \\
& \simeq-\int 3 H^{2} \frac{d \phi_{j}}{V_{j}^{\prime}\left(\phi_{j}\right)} \\
& \simeq-\frac{1}{M_{p}^{2}} \int\left(\sum_{i} V_{i}\left(\phi_{i}\right)\right) \frac{d \phi_{j}}{V_{j}^{\prime}\left(\phi_{j}\right)} \\
& =-\frac{1}{M_{p}^{2}} \sum_{i} \int_{\phi_{i}^{*}}^{\phi_{i}^{e}} \frac{V_{i}}{V_{i}^{\prime}} d \phi_{i},
\end{aligned}
$$


where Eqs. (2), (3) and (44) have been used, and the upper limit $\phi_{i}^{e}$ of the integral corresponds to the end of inflation and "*" denotes the horizon-crossing time of corresponding perturbation and generally $\phi_{i}^{*} \lesssim \phi_{i}^{e}$.

From Eq. (11), we have

$$
\frac{V_{i}}{V_{i}^{\prime}} \simeq \frac{1}{p}\left[\phi_{i}-\frac{\mu_{i}^{p}}{\phi_{i}^{p-1}}\right]
$$

Substituting (6) into (5) and after simplification, we get

$$
N \simeq \frac{1}{p M_{p}^{2}} \sum_{i}\left[\frac{1}{2}\left(\phi_{i}^{* 2}-\phi_{i}^{e 2}\right)+\frac{\mu_{i}^{p}}{p-2}\left[\frac{1}{\phi_{i}^{* p-2}}-\frac{1}{\phi_{i}^{e p-2}}\right]\right] .
$$

Inflation ends at $\phi_{i}^{e} \lesssim \mu_{i}$, and in the meantime in the small-field model we have $\mu_{i} \lesssim M_{p}$, thus the quadratic terms which appear in Eq. (17) must vanish since $\phi_{i}^{*} \lesssim \phi_{i}^{e} \lesssim M_{p}$, i.e. $\left(\frac{\phi_{i}^{*}}{M_{p}}\right)^{2} \rightarrow 0,\left(\frac{\phi_{i}^{e}}{M_{p}}\right)^{2} \rightarrow 0$. Thus we have

$$
N \simeq \frac{1}{p(p-2) M_{p}^{2}} \sum_{i}\left(\frac{\mu_{i}}{\phi_{i}^{*}}\right)^{p-2}\left[1-\left(\frac{\phi_{i}^{*}}{\phi_{i}^{e}}\right)^{p-2}\right] \mu_{i}^{2}
$$

Further by taking $1-\left(\frac{\phi_{i}^{*}}{\phi_{i}^{e}}\right)^{p-2} \bumpeq 1$, Eq. (8) is reduced to

$$
N \simeq \frac{1}{p(p-2) M_{p}^{2}} \sum_{i}\left(\frac{\mu_{i}}{\phi_{i}^{*}}\right)^{p-2} \mu_{i}^{2}
$$

Generally for multi-field there exists not only the curvature perturbation, but also the orthogonal isocurvature perturbations [22, 23], see [24] for a review. To simplicity, we assume here that the isocurvature perturbation may be neglected, which will be further discussed in section 4. In this case, the curvature perturbation of multi-field inflation can be evaluated analytically by using the Sasaki -Stewart formulism [25] also earlier in Ref. [26]. Thus the spectral index is given by

$$
n_{s}-1 \simeq-M_{p}^{2} \frac{\sum_{i}\left(V_{i}^{\prime}\right)^{2}}{V^{2}}-2 M_{p}^{2} \frac{1}{\sum_{i}\left(\frac{V_{i}}{V_{i}^{\prime}}\right)^{2}}+2 M_{p}^{2} \frac{1}{V} \frac{\sum_{i}\left(\frac{V_{i}}{V_{i}^{\prime}}\right)^{2} V_{i}^{\prime \prime}}{\sum_{j}\left(\frac{V_{j}}{V_{j}^{\prime}}\right)^{2}} .
$$

To make the relevant formula clear, we introduce a suitable substitution for summation terms. We introduce

$$
A_{1}=\sum_{i}\left(w_{i}\right)^{p-2} \mu_{i}^{2}
$$

where $w_{i}=\mu_{i} / \phi_{i}^{*}$. Substituting Eq. (11) into Eq. (9), the efolding number can be rewritten as

$$
N \simeq \frac{A_{1}}{p(p-2) M_{p}^{2}}
$$

which implies that

$$
M_{p}^{2} \simeq \frac{A_{1}}{p(p-2)} \frac{1}{N}
$$

Then we calculate all terms in right side of Eq. (10) separately,

$$
\begin{gathered}
V^{2} \simeq\left[\sum_{i} \Lambda_{i}\right]^{2} \equiv A_{2}^{2}, \\
\sum_{i}\left(V_{i}^{\prime}\right)^{2}=p^{2} \sum_{i} \frac{\Lambda_{i}^{2}}{\left(w_{i}\right)^{2(p-1)} \mu_{i}^{2}} \equiv p^{2} A_{3},
\end{gathered}
$$




$$
\begin{gathered}
\sum_{i}\left(\frac{V_{i}}{V_{i}^{\prime}}\right)^{2} \simeq \frac{1}{p^{2}} \sum_{i}\left(w_{i}\right)^{2(p-1)} \mu_{i}^{2} \equiv \frac{A_{4}}{p^{2}} \\
\sum_{i}\left(\frac{V_{i}}{V_{i}^{\prime}}\right)^{2} V_{i}^{\prime \prime} \simeq-\frac{(p-1)}{p} \sum_{i} \Lambda_{i}\left(w_{i}\right)^{p} \equiv-\frac{(p-1)}{p} A_{5} .
\end{gathered}
$$

Substituting the above Eqs. into Eq. (10), we have the scalar spectral index

$$
n_{s}-1 \simeq-\frac{2}{N}\left(\frac{p-1}{p-2}\right)\left[\frac{p}{2(p-1)}\left(\frac{A_{1} A_{3}}{A_{2}^{2}}\right)+\frac{p}{p-1}\left(\frac{A_{1}}{A_{4}}\right)+\frac{A_{1} A_{5}}{A_{2} A_{4}}\right],
$$

where Eq. (13) has been used. Note that $\left(1 / w_{i}^{2(p-1)}\right) \simeq 0$, and $1 / w_{i}^{p} \simeq 0$, thus the first and second terms on right hand side of Eq. (18) must vanish, i.e. $\frac{p}{2(p-1)}\left(\frac{A_{1} A_{3}}{A_{2}^{2}}\right) \simeq 0$, and $\frac{p}{p-1}\left(\frac{A_{1}}{A_{4}}\right) \simeq 0$. Then we get the spectral index

$$
n_{s}-1 \simeq-\frac{2}{N} \frac{p-1}{p-2}\left[1+R\left(w_{i}\right)\right]
$$

where

$$
R\left(w_{i}\right)=\frac{\left(A_{1} A_{5}-A_{2} A_{4}\right)}{A_{2} A_{4}} .
$$

Thus with the definitions of $A_{1}, A_{2}, A_{4}$ and $A_{5}$, we have

$$
R\left(w_{i}\right)=\frac{\sum_{i<j}\left(w_{j}^{p}-w_{i}^{p}\right)\left(\Lambda_{j} w_{i}^{p-2} \mu_{i}^{2}-\Lambda_{i} w_{j}^{p-2} \mu_{j}^{2}\right)}{\sum_{i, j} \Lambda_{i} w_{j}^{2 p-2} \mu_{j}^{2}} .
$$

The mass $m_{i}^{2}$ of each scalar field $\phi_{i}$ can be defined as

$$
m_{i}^{2}=V_{i}^{\prime \prime}=-p(p-1) \Lambda_{i} \frac{1}{w_{i}^{p-2} \mu_{i}^{2}} .
$$

Thus by substituting Eq. (22) into Eq. (21), we can rewrite $R\left(w_{i}\right)$ as

$$
R\left(w_{i}\right)=\frac{\sum_{i<j} \Lambda_{i} \Lambda_{j}\left(w_{j}^{p}-w_{i}^{p}\right)\left(\frac{1}{m_{i}^{2}}-\frac{1}{m_{j}^{2}}\right)}{\sum_{i, j} \Lambda_{i} \Lambda_{j} w_{j}^{p} / m_{j}^{2}} .
$$

The first term in the right hand side of Eq. (19) is just the result of single field inflation [11, while the second term is additional term which appears due to multi-field as shown in Eq. (23). When all fields have same masses at the horizon-crossing time, we have $R\left(w_{i}\right)=0$, and thus

$$
n_{s}-1 \simeq-\frac{2}{N}\left(\frac{p-1}{p-2}\right) .
$$

In this case the scalar spectrum of multi-field will be the same as that of its corresponding single field [11. When $w_{1}<w_{2}<w_{3} \ldots<w_{n}$ and $m_{1}^{2}<m_{2}^{2}<\ldots<m_{n}^{2}, R\left(w_{i}\right)$ will be always positive, which means that spectrum is more redder than that of its corresponding single field. But when $w_{1}<w_{2}<w_{3} \ldots<w_{n}$ and $m_{1}^{2}>m_{2}^{2}>\ldots>m_{n}^{2}, R\left(w_{i}\right)$ will be always negative, which means that the spectrum is less red than that of its corresponding single field. However, for more general cases, it seems that dependent of parameters of fields and initial conditions, there is no definite conclusion.

In the case of two scalar fields, from Eq. (23), we can get

$$
R\left(w_{1}, w_{2}\right)=\frac{\Lambda_{1} \Lambda_{2}\left(w_{2}^{p}-w_{1}^{p}\right)\left(1 / m_{1}^{2}-1 / m_{2}^{2}\right)}{\left(\Lambda_{1}+\Lambda_{2}\right)\left(\Lambda_{1} w_{1}^{p} / m_{1}^{2}+\Lambda_{2} w_{2}^{p} / m_{2}^{2}\right)} \simeq \frac{\left(w_{2}^{p}-w_{1}^{p}\right)\left(m_{2}^{2}-m_{1}^{2}\right)}{\left(1 / \Lambda_{2}+1 / \Lambda_{1}\right)\left(\Lambda_{1} m_{2}^{2} w_{1}^{p}+\Lambda_{2} m_{1}^{2} w_{2}^{p}\right)},
$$

if $w_{1}<w_{2}$ and $m_{1}^{2}<m_{2}^{2}, R\left(w_{1}, w_{2}\right)$ will be positive, which implies that the scalar spectrum is more red than that of its corresponding single field, but if $w_{1}<w_{2}$ and $m_{1}^{2}>m_{2}^{2}, R\left(w_{1}, w_{2}\right)$ will be negative, which implies that the scalar spectrum is less red than that of its corresponding single field. 


\section{The Scalar Spectrum for $p=2$}

The Eq. (19) is only valid for $p>2$, so we need to separately calculate the case of $p=2$ which will be done in this section. We consider a general potential as $V=\sum_{i} \Lambda_{i}\left[1-\left(\frac{\phi_{i}}{\mu_{i}}\right)^{2}\right]$. In this case $V_{i}^{\prime}=-2 \Lambda_{i} \frac{\phi_{i}}{\mu_{i}^{2}}$ and $V_{i}^{\prime \prime}=-2 \Lambda_{i} \frac{1}{\mu_{i}^{2}}$. Moreover, we can approximate $\left(\frac{\phi_{i}^{*}}{M_{p}}\right)^{2} \longrightarrow 0$, $\left(\frac{\phi_{i}^{e}}{M_{p}}\right)^{2} \longrightarrow 0$. Thus, following the same steps as we did in the previous section, we obtain

$$
N \simeq-\frac{1}{2 M_{p}^{2}} \sum_{i} \ln \left(\frac{\phi_{i}^{*}}{\phi_{i}^{e}}\right) \mu_{i}^{2}
$$

From Eq. (10), we get the spectral index

$$
n_{s}-1 \simeq-2 M_{p}^{2}\left[2 \frac{\sum_{i}\left(\Lambda_{i}^{2} / \mu_{i}^{2} w_{i}^{2}\right)}{\left(\sum_{i} \Lambda_{i}\right)^{2}}+4 \frac{1}{\sum_{i} \mu_{i}^{2} w_{i}^{2}}+2 \frac{\sum_{i} \Lambda_{i} w_{i}^{2}}{\sum_{i} \Lambda_{i} \sum_{j} \mu_{j}^{2} w_{j}^{2}}\right] .
$$

Note that $1 / w_{i}^{2} \simeq 0$, thus the first two terms on right side of above equation can be neglected, which reduces Eq. (27) to

$$
n_{s}-1 \simeq-4 M_{p}^{2} \frac{\sum_{i} \Lambda_{i} w_{i}^{2}}{\sum_{i} \Lambda_{i} \sum_{j} \mu_{j}^{2} w_{j}^{2}}
$$

From Eq. (4), we have

$$
\frac{\dot{\phi}_{i}}{\dot{\phi}_{j}} \simeq \frac{\Lambda_{i} \phi_{i} / \mu_{i}^{2}}{\Lambda_{j} \phi_{j} / \mu_{j}^{2}}
$$

which implies that

$$
\mu_{i}^{2} \frac{\dot{\phi}_{i}}{\Lambda_{i} \phi_{i}} \simeq \mu_{j}^{2} \frac{\dot{\phi}_{j}}{\Lambda_{j} \phi_{j}}
$$

By taking the integral on both side of Eq. (30), we get

$$
\frac{\mu_{i}^{2}}{\Lambda_{i}} \ln \left(\frac{\phi_{i}^{*}}{\phi_{i}^{e}}\right)=\frac{\mu_{j}^{2}}{\Lambda_{j}} \ln \left(\frac{\phi_{j}^{*}}{\phi_{j}^{e}}\right)
$$

which implies that

$$
\sum_{i} \mu_{i}^{2} \ln \left(\frac{\phi_{i}^{*}}{\phi_{i}^{e}}\right)=\mu_{k}^{2} \ln \left(\frac{\phi_{k}^{*}}{\phi_{k}^{e}}\right) \sum_{i} \frac{\Lambda_{i}}{\Lambda_{k}} \quad \text { by taking } j=k,
$$

It should be noted that for the fixed value of " $\mathrm{k}$ " we get relation (32), in which the term with subscript " $k$ " is independent of summation notation. Now substituting Eq. (32) into Eq. (26), we obtain

$$
M_{p}^{2} \simeq-\frac{1}{2 N} \mu_{k}^{2} \ln \left(\frac{\phi_{k}^{*}}{\phi_{k}^{e}}\right) \sum_{i} \frac{\Lambda_{i}}{\Lambda_{k}} .
$$

which then is substituted into Eq. (28) and gives

$$
n_{s}-1 \simeq \frac{2}{N} \ln \left(\frac{\phi_{k}^{*}}{\phi_{k}^{e}}\right) \frac{\sum_{i} w_{i}^{2}\left(\frac{\Lambda_{i}}{\Lambda_{k}}\right)}{\sum_{j}\left(\mu_{j}^{2} / \mu_{k}^{2}\right) w_{j}^{2}} .
$$

If all fields have same $\Lambda_{i}$, we will obtain

$$
n_{s}-1 \simeq \frac{2}{N} \ln \left(\frac{\phi_{k}^{*}}{\phi_{k}^{e}}\right) \frac{\sum_{i} w_{i}^{2}}{\sum_{j}\left(\mu_{j}^{2} / \mu_{k}^{2}\right) w_{j}^{2}} .
$$


The results of Eqs. (34) and (35) are strictly independent of the choice of $k$. This can be seen as follows. For example, we take $k=k_{1}$ and $k=k_{N}$, and put them into Eq. (35). If we call the corresponding indices $n_{k_{1}}-1$ and $n_{k_{N}}-1$ respectively, then $\left(n_{k_{1}}-1\right) /\left(n_{k_{N}}-1\right)$ is simply 1 because of Eq. (31) with $\Lambda_{i}$ being the same for all $i$.

Further, if all fields have same $\mu_{i}$, we will have

$$
n_{s}-1 \simeq \frac{2}{N} \ln \left(\frac{\phi_{k}^{*}}{\phi_{k}^{e}}\right) .
$$

Note that in this case all $\ln \left(\frac{\phi_{i}^{*}}{\phi_{i}^{e}}\right)$ are equivalent due to Eq. (31). Eq.(36) is actually same as with that for its corresponding single field.

Now let us see Eq. (35). If all $\Lambda_{i}$ are taken to be same while $\mu_{i}$ are different, from Eq. (31), it is obvious that a larger $\mu_{i}$ will correspond to a smaller $\ln \left(\frac{\phi_{i}^{*}}{\phi_{i}^{e}}\right)$. When taking $\mu_{k}=$ $\operatorname{Max}\left(\mu_{1}, \mu_{2}, \ldots \mu_{n}\right)$, which implies that $\mu_{i} / \mu_{k}<1$, from Eq. (35)), we have the spectrum is more red than that of single field $\phi_{k}$, i.e. Eq. (36). Note that in this case $\ln \left(\frac{\phi_{k}^{*}}{\phi_{k}^{e}}\right)$ is the smallest in all $\ln \left(\frac{\phi_{i}^{*}}{\phi_{i}^{e}}\right)$, which suggests that in term of Eq. (36), the spectrum for single field $k$ is the nearest from 1. When taking $\mu_{k}=\operatorname{Min}\left(\mu_{1}, \mu_{2}, \ldots \mu_{n}\right)$, which implies that $\mu_{i} / \mu_{k}>1$, from Eq. (35), we have the spectrum is less red than that of single field $\phi_{k}$. Note that in this case $\ln \left(\frac{\phi_{k}^{*}}{\phi_{k}^{e}}\right)$ is the largest in all $\ln \left(\frac{\phi_{i}^{*}}{\phi_{i}^{e}}\right)$. The above results means that for $p=2$ the scalar spectral index generally lies between that of single field with the largest $\mu_{k}$ and that of single field with the smallest $\mu_{k}$.

\section{Conclusion}

We have studied the spectrum of curvature perturbation of multi-field inflation with general small-field potential. For $p>2$, we found that the spectrum may be redder or bluer than that of its corresponding single field. The result is depending on the value of fields and their effective masses at the horizon-crossing time. This result is different from that of multi-field inflation with power law potential, in which the definite conclusion that the spectrum is redder than that of its corresponding single field may be obtained. When the effective masses of all fields are equal, the spectrum will be the same with its corresponding single field. The behavior for $p=2$ is different from that of $p>2$. In this case we observed that the scalar spectral index generally lies between that of single field with the largest $\mu_{k}$ and that of single field with the smallest $\mu_{k}$. When all $\mu_{i}$ are taken to be equivalent, the spectrum will be the same with that of its single field.

In our study, the results are dependent of not the initial conditions, but the values of fields and their effective masses at the horizon-crossing time, since the perturbation spectrum is calculated at the horizon-crossing time of corresponding perturbation, which in fact is a good approximation, as was discussed in Ref. [13. In order to obtain this result, we only require that the slow roll approximation for each field is satisfied initially and also at all time.

It should be noted that here we have assumed that the isocurvature perturbation may be neglected. In fact, in order to neglect the isocurvature perturbation, the conditions $\delta \phi_{i} / \dot{\phi}_{i}=$ $\delta \phi_{j} / \dot{\phi}_{j}$ have to be imposed. In this case, it seems that the fields must be constrained in some special trajectories. In addition, it is also possible that for general trajectories at some special horizon-crossing time of corresponding perturbation, the above condition is just satisfied. The results here are actually suitable for above both cases. Though in general case the inclusion of isocurvature mode seems required, such study for general large $\mathrm{N}$ fields is beyond our ability at present. However, our work may be regarded as one interesting step along this line. 


\section{Acknowledgment}

I.A would like to thanks the support of (HEC)Higher Education Commission of Pakistan in favor of research in China. This work is supported in part by the National Natural Science Foundation of China (NSFC) No: 10491306, 10521003, 10775179, 10405029, 10775180, in part by the Scientific Research Fund of GUCAS(NO.055101BM03), in part by CAS under Grant No: KJCX3-SYW-N2.

\section{References}

[1] A.H. Guth, Phys. Rev. D23 (1981) 347.

[2] A.D. Linde, Phys. Lett. B108 (1982) 389; A.A. Albrecht and P.J. Steinhardt, Phys. Rev. Lett. 48 (1982) 1220.

[3] For an earlier attempt of inflation model, see A. A. Starobinsky, Phys. Lett. B91, 99 (1980).

[4] A.R. Liddle, A. Mazumdar, and F.E. Schunck, Phys. Rev. D58, 061301 (1998).

[5] K.A. Malik and D. Wands, Phys. Rev. D59, 123501 (1999); E.J. Copeland, A. Mazumdar and N.J. Nunes, Phys. Rev. D60, 083506 (1999); A.M. Green and J.E. Lidsey, Phys. Rev. D61, 067301 (2000); A. Mazumdar, S. Panda and A. Perez-Lorenzana, Nucl. Phys. B614, 101 (2001).

[6] P. Kanti and K.A. Olive, Phys. Rev. D60, 043502 (1999); Phys. Lett. B464, 192 (1999); N. Kaloper and A.R. Liddle Phys. Rev. D61, 123513 (2000).

[7] Y.S. Piao, W. Lin, X. Zhang and Y.Z. Zhang, Phys. Lett. B528, 188 (2002).

[8] Y.S. Piao, R.G Cai, X. Zhang and Y.Z. Zhang Phys. Rev. D66, 121301 (2002); M. Majumdar and A.C. Davis Phys. Rev. D69, 103504 (2004); R. Brandenberger, P. Ho and H. Kao, JCAP 0411, 011 (2004) ; A. Jokinen and A. Mazumdar, Phys. Lett. B597, 222 (2004); K. Becker, M. Becker and A. Krause, Nucl. phys. B715 (2005) 349-371; J.M. Cline and H. Stoica, Phys. Rev. D72, 126004 (2005); J. Ward, Phys. Rev. D73, 026004 (2006).

[9] D. Seery and J.E. Lidsey, JCAP 0509 (2005) 011; G.I. Rigopoulos, E.P. S. Shellard and B.W. Van, Phys. Rev. D76, 083512 (2007); R. Easther and J.T. Giblin, phys. Rev. D72, 103505 (2005).

[10] S. Dimopoulos, S. Kachru, J. McGreevy and J. Wacker, arXiv:hep-th/0507205.

[11] L. Alabidi, and D.H. Lyth, JCAP 0605, 016 (2006).

[12] R. Easther and L. McAllister, JCAP 0605, 018 (2006).

[13] S.A. Kim and A.R. Liddle, Phys. Rev. D74, 023513 (2006).

[14] Y.S. Piao, Phys. Rev. D74, 047302 (2006).

[15] J.O. Gong, Phys. Rev. D75, 043502 (2007); M.E. Olsson, JCAP 0704, 019 (2007); S. Thomas, J. Ward, Phys. Rev. D76, 023509 (2007).

[16] S.A. Kim and A.R. Liddle, Phys. Rev. D74, 063522 (2006); Phys. Rev. D76, 063515 (2007).

[17] A.D. Linde, Phys. Lett. B108, 389 (1982). 
[18] A. Albrecht and P.J. Steinhardt, Phys. Rev. Lett. 48, 1220 (1982).

[19] K. Dimopoulos and G. Lazarides, Phy. Rev. D73, 023525 (2005).

[20] K. Freese, J. Frieman and A. Olinto, Phys. Rev. Lett. 65, 3233 (1990); L. Randall, JCAP 0307, 003 (2003); K.I. Izawa, M. Kawasaki and T. Yanagida, Phys. Lett. B411, 249 (1997); J.A. Adams, G.G. Ross and S. Sarkar, Phys. Lett. B391, 271 (1997).

[21] D.H. Lyth and A.Ritto, Phys. Rept. 314, (1999) 1-146.

[22] D. Langlois, Phys. Rev. D59, 123512 (1999).

[23] C. Gordon, D. Wands, B.A. Bassett and R. Maartens, Phys. Rev. D63, (2001) 023506; L. Amendola, C. Gordon, D. Wands and M. Sasaki, Phys. Rev. Lett. 88, 211302 (2002); C.T. Byrnes and D. Wands, Phys. Rev. D74, (2006) 043529.

[24] B.A. Bassett, S. Tsujikawa and D. Wands, Rev. Mod. Phys. 78 (2006) 537.

[25] M. Sasaki and E.D. Stewart, Prog. Theor. Phys. 95, 71 (1996).

[26] A.A. Starobinsky, JETP Lett. 42, 152 (1985). 